\title{
TENSOR PRODUCTS OF SEPARABLE REDUCED PRIMARY MODULES
}

\author{
by CARY WEBB \\ (Received 21st June 1976, revised 1st July 1977)
}

$\boldsymbol{R}$ will denote a Dedekind domain and Pone of its prime ideals. A $P$-primary module will be an $R$-module all of whose non-zero elements have annihilators that are powers of the prime $P$. In all that follows $E$ is such a module.

The height of $0 \neq x \in E$ will be $\max \left\{n: x \in P^{n} E\right\}$. It is denoted by $h(x)$. If this maximum does not exist we will say $h(x)=\infty$.

Clearly the condition $\cap_{n=1}^{\infty} P^{n} E=0$ is equivalent to $E$ having no non-zero elements of infinite height. Adopting the terminology of (2, Ch. XI) where such modules over the ring of integers are studied, we will call these modules separable and reduced.

We remark that separability, as it was originally defined (1), referred to the property of each finite subset being contained in a direct summand that is itself a finite direct sum of rank 1 submodules. (Actually, Baer restricted himself to torsion-free groups.) In the case of reduced $P$-primary modules, being separable in this sense and not having elements of infinite height are equivalent properties. Simple examples (e.g., the field quotients) show that this does not hold for arbitrary modules.

In this study it is our purpose to prove that among reduced $P$-primary modules, separable modules $E$ are characterised by the property that for any family of (mixed) $R$-modules $\left(F_{i}\right)_{i \in I}$ the canonical homomorphism $f: E \bigotimes_{R} \Pi_{i \in I} F_{i} \rightarrow \Pi_{i \in I}\left(E \bigotimes_{R} F_{i}\right)$ that takes a generator $x \otimes\left(y_{i}\right)_{i \in I} \in E \otimes_{R} \Pi_{i \in I} F_{i}$ onto $\left(x \otimes y_{i}\right)_{i \in I} \in \Pi_{i \in I}\left(E \otimes_{R} F_{i}\right)$ is injective. We shall also show that this embedding is pure, that is, if id: $G \rightarrow G$ is the identity on an arbitrary $R$-module $G$, then $f \otimes$ id is injective.

For convenience we will omit writing the indexing set $I$ whenever there is no possibility of confusion. Instead of writing $E \otimes_{R} F$ we will simply write $E \otimes F$. Often an obvious homomorphism will not be defined explicitly or even named.

This paper continues an investigation begun in (5). The reader is referred to (5) for more background and further facts about the homomorphism $f$. (6) and (7) contain an application of our knowledge of $f$ to the study of the natural transformation of abelian groups, hom $(A, B) \otimes C \rightarrow \operatorname{hom}(\operatorname{hom}(C, A), B)$.

Theorem 1. A reduced $P$-primary module $E$ is separable iff for every family of $R$-modules $\left(F_{i}\right)$, the natural homomorphism $E \otimes \Pi F_{i} \rightarrow \Pi\left(E \otimes F_{i}\right)$ is injective.

Proof. Suppose $E$ is separable and let $\left(F_{i}\right)$ be an arbitrary family of $\boldsymbol{R}$-modules. Suppose $z=\sum_{k=1}^{n} x_{k} \otimes\left(y_{i k}\right) \in E \otimes \Pi F_{i}$ and $f(z)=\sum_{k=1}^{n}\left(x_{k} \otimes y_{i k}\right)_{i \in I}=0$. By (3, Lemma 